\title{
THE PHOTOGRAPHIC EMULSION: SENSITIZATION BY SODIUM SULPHITE
}

\author{
By Burt H. Carroll and Donald Hubbard
}

\section{ABSTRACT}

Sodium sulphite, in quantities sufficient to reduce a few tenths percent of the silver bromide of a photographic emulsion, may act as a powerful sensitizer only slightly inferior to the natural sensitizers in gelatin. It acts as such only after digestion with the emulsion, insuring that it has reacted with the silver bromide. The sensitivity nuclei thus formed are of metallic silver, in amounts similar to the silver sulphide nuclei of normal emulsions. The rate of change of sensitivity increases with increasing alkalinity and decreases with increasing bromide-ion concentration. Sulphite may be added to the emulsion before washing, sensitizing only on digestion after washing. There was no indication of panchromatic sensitization (Capstaff effect).

\section{CONTENTS}

I. Introduction

II. Experimental

1. The reaction between sodium sulphite and excess silver bromide

2. Preparation of experimental emulsions

3. Sensitometry

4. Spectral sensitivity of sulphite-sensitized emulsions

5. The effect of concentration of sensitizer

6. Fog and stability of sulphite-sensitized emulsions

7. Hydrogen-ion concentration

8. Bromide-ion concentration

9. Emulsions made with sulphite added before washing

10. Experiments with other reducing agents

III. Conclusions

\section{INTRODUCTION}

It has long been known that if pure silver bromide is precipitated from water solutions and subsequently suspended in gelatin, the resulting photographic emulsion is very insensitive. While there are difficulties connected with the emulsification under these conditions, it suggested that the grains of an emulsion may not be pure silver bromide (or bromide-iodide). Further evidence was supplied by Lüppo-Cramer's discovery ${ }^{1}$ (1), later extended by Clark, (2), (3), (4), and others, that the sensitivity of ripened emulsions was greatly reduced by treatment with oxidizing agents, such as chromic acid, which do not attack silver bromide. Statistical studies of the sensitivity of individual grains and of the distribution of the development

\footnotetext{
${ }^{1}$ Numbers in parentheses here and throughout the text refer to the list of references at the end of the paper.
} 
centers on them (5) indicated that it is highly probable that the unexposed grains of an emulsion contain nuclei which are different from the rest of the grain, and a most important factor in sensitivity. Finally, Sheppard and his coworkers (6), (7) demonstrated the existence of materials which act as powerful sensitizers for emulsions when used under conditions which insure the formation of a trace of silver sulphide by reaction between silver bromide and the sensitizer.

The hypothesis naturally suggested by the desensitizing action of oxidizing agents was that traces of silver bromide had been reduced during the ripening process to silver. It has never been definitely proved or disproved that the nuclei of ripened emulsions contain metallic silver. Clark (8) attempted to distinguish between silver and silver sulphide by means of oxidizing agents of varying potential, without conclusive results. Weigert and Lühr (9) believed that they had proved by treatment with persulphate that the "Ursilber" (silver not combined with halogen) could not be silver sulphide, but this has since been found (10), (11) to be incorrect. On the other hand, Carroll and Hubbard (12) demonstrated that colloidal silver may, under special conditions, sensitize an emulsion, apparently by functioning as nuclei after adsorption to the grains. In this paper it is shown that sodium sulphite may have photographic-sensitizing action approaching that of allyl thiocarbamide, when it is added to emulsions under such conditions that it must reduce traces of the silver bromide to metal. The hypothesis that metallic silver may act as sensitivity nuclei, therefore, appears to be proved. Reduction of the silver bromide before exposure normally causes fog, exactly analogous to the fog produced by the formation of silver sulphide from inorganic sulphides. This turns out to be fresh proof of the fact that has become increasingly evident (14) lately, that the quantity of nonhalide silver in an emulsion is much less important than what we may call its quality for want of more definite knowledge. Sheppard (6), (15) has observed that all the sulphur, selenium, and tellurium sensitizing compounds specified by him form complexes with silver halides, and that no sensitization is produced by equivalent amounts of inorganic sulphides. Sodium sulphite is closely analogous. Difficultly soluble silver salts may be dissolved in sulphite solutions by formation of a complex ion, probably $\mathrm{Ag}\left(\mathrm{SO}_{3}\right)_{2}{ }^{-}$, similar to that formed by thiosulphate or thiocarbamide. The mechanism of reduction in one case and sulphide formation in the other is probably the same. This will be discussed in a separate paper on the kinetics of these reactions.

One hypothesis which cannot be excluded on the present evidence is that the presence of both silver and silver sulphide is essential to sensitization. Sheppard and Punnett (16) have patented the use of reducing agents such as sodium "hydrosulphite" (but not sulphite) in combination with sensitizers giving silver sulphide, and Trivelli has elaborated a theory (17) of sensitivity and the latent image based on a compound nucleus. Under the conditions of the experiments to be described in this paper, silver should have been formed in proportions greater than silver sulphide, but no gelatin emulsions can be proved to be free from the latter. Some light might be thrown on this point by testing the sensitizing action of sulphite on emulsions made up in gum arabic known to be free from labile sulphur.

The addition of sulphite to photographic emulsions is by no means new; the novelty of our results depends on the conditions of its use. 
The earliest references (13) are to the combined use of sulphite and ammonia, which caused so much reduction that a mirror was deposited on the walls of the vessel, with consequent fog. Valenta (18) digested Lippmann emulsions with $1 \mathrm{~g} \mathrm{Na} \mathrm{Na}_{2} \mathrm{SO}_{3}$ to $300 \mathrm{~g}$ emulsion at $38^{\circ}$. The sulphite retarded growth of grain; plates ripened up to 30 minutes could be used for the Lippmann process, while without it, the grain grew enough to give duller colors. Emulsions which were $4^{\circ}$ Warneke after 5 minutes digestion with the sulphite were $18^{\circ}$ after 1 hour digestion. Lüppo-Cramer (19) reports that bromine absorbents such as sulphite may increase the sensitivity of Lippmann emulsions, but the evidence in the case of Valenta's work is that the sensitization was the result of reaction of the sulphite and silver bromide, since prolonged digestion was necessary for the full effect. This feature of Valenta's work has escaped notice, although the statement about the grain size has not; in the one recent reference (20) to the use of sulphite in an emulsion formula, Valenta's experiments are said to have inspired the idea of adding sulphite to produce fine grain and high contrast.

Collodial silver in emulsions has frequently been observed to cause spectral sensitization; this effect, which has recently been reviewed by Sheppard (22), is well established, while general sensitization by silver has remained a matter of conjecture. Silver sulphide is capable of producing both effects-general sensitization when materials such as allyl thiocarbamide are added to the emulsion before digestion, and spectral sensitization when plates are bathed in the same materials, or when silver bromide and colloidal silver sulphide are precipitated together to form the analogue of the photobromide. In this case, Sheppard has concluded that the spectral sensitization is caused by larger numbers of very fine silver-sulphide particles, while the general sensitization is caused by fewer and coarser nuclei. Similar results are to be expected with silver. The analogy is apparently complete, since we have obtained general sensitization by adding sulphite to the emulsion before digestion, while Capstaff and Bullock (21) found that some emulsions (Eastman Portrait film) acquired panchromatic sensitivity by bathing in solutions of sodium bisulphite and washing in dilute alkali. The alkali was necessary to the sensitization, while soluble bromide prevented it. It was accompanied by visible discoloration of the emulsion. All this evidence indicates that the silver halide of the emulsion was reduced by the sulphite, so that the sensitization may be attributed to colloidal silver.

Investigation of photographic emulsions at this bureau has been suspended for lack of funds. The experimental data here presented are admittedly incomplete in some respects, which we have attempted to indicate, but the results seem to be quite adequate to justify publication.

\section{EXPERIMENTAL}

1. THE REACTION BETWEEN SODIUM SULPHITE AND EXCESS SILVER BROMIDE

Data on the kinetics of the reduction of silver bromide by dilute solutions of sodium sulphite will be given in a separate communication. The reaction may be written $2 \mathrm{AgBr}+\mathrm{Na}_{2} \mathrm{SO}_{3}+\mathrm{H}_{2} \mathrm{O}=$ $2 \mathrm{Ag}+2 \mathrm{NaBr}+\mathrm{H}_{2} \mathrm{SO}_{4}$. It is retarded 'by the products, hydrogen and bromide ions. Under the conditions of sensitization in the emulsion, which involve reduction of not over 0.01 percent of the silver 
bromide, the change in concentration of these ions produced by the reaction itself is negligible.

While nothing was found in the literature to indicate that there might be side reactions, the known tendency of solutions of sulphur dioxide to form thiosulphuric and polythionic acids made it essential to test the products of the reaction for silver sulphide. Silver bromide or chloride was boiled with sodium sulphite solution, the former being in excess. The solid reaction products were oxidized with concentrated nitric acid. No sulphate was found after this treatment, although it would have been determined if as little as 0.1 percent of the silver reacting had gone to sulphide.

As the tendency for rearrangement of sulphite solutions is greatest at the boiling point, these experiments should have been an adequate test and we may say that the sensitizing action of sodium sulphite on emulsions cannot be explained by formation of silver sulphide.

The sodium sulphite used in the experiments was a c.p. product of unusually high grade, assaying 99 percent $\mathrm{Na}_{2} \mathrm{SO}_{3}$ by iodine titration. It was tested for the presence of thiosulphates or polythionates by the sensitive azide method of Feigl (24) with completely negative results. Stock solutions, about tenth normal, were kept under hydrogen, maintaining their titer unchanged for over a week.

The hypothesis that the sulphite may act simply as a bromine absorbent is satisfactorily eliminated by the complete absence of any immediate effect on sensitivity. In two cases where marked sensitizing action was developed on subsequent digestion with the sulphite (emulsions 4-124 and 125, fig. 4), the emulsion directly after its addition was, within the limits of error, no different from the controls. Using the speed number $10 / E_{m}$ (for 6-minute development) in one case the control was 11, the batch with sulphite 10 ; in the other case the figures were 10 and 10.5. The sulphite-sensitized portions developed speeds of 1,400 and 300, on the same basis, on subsequent digestion.

\section{PREPARATION OF EXPERIMENTAL EMULSIONS}

The general methods of making experimental emulsions in this laboratory have been described in other papers. The formulas used in the experiments where sulphite was added after washing will be given in full at this point; some of the experiments with sulphite in the original mix of the emulsion will be described in a later section. Practically all the emulsions were of the neutral type. The sensitizing effect of sulphite was first tested by adding sodium sulphite or sulphur dioxide solutions to washed emulsions which had been made by the ammonia process, but no experiments have been made by adding sulphite to the original solutions of an ammonia-process emulsion. It is probable that sensitization could be produced in this way, but the sulphite would be rapidly oxidized by the air under these conditions and difficulty in reproducing results is to be anticipated.

All the emulsions were washed by the conventional method of shredding the set jelly and extracting with cold water. Except for a few otherwise noted, they were washed in changes of distilled water plus $1 \frac{1}{4} \mathrm{~g} \mathrm{MgSO}_{4} \cdot 7 \mathrm{H}_{2} \mathrm{O}$ per liter, using the apparatus described in a recent note (25). This has been improved by the addition of a 
stirring device. A cam rotating about three times a minute, slowly lifts the nickel-gauze basket containing the emulsion $4 \mathrm{~cm}$, then drops it suddenly through the water. This has more than doubled the rate of washing; there is very little loss of emulsion since the noodles are not scraped against the wire gauze as by rotary stirring.

The formula most used has already been described under the designation of " $\mathrm{C}$ " in a previous paper (27); it will be referred to as $\mathrm{C}-1$ in this case. The solutions are:

$\mathrm{NH}_{4} \mathrm{Br}\left(0.429 \mathrm{~g}\right.$ equivalent) _- $42.0 \mathrm{~g} \mid \mathrm{AgNO}_{3}(0.353 \mathrm{~g}$ equivalent $) \ldots \quad 60.0 \mathrm{~g}$ KI $(0.0139 \mathrm{~g}$ equivalent) _... $2.30 \mathrm{~g}$

Gelatin

Water $450 \mathrm{ml}$

Water 20

$\mathrm{g}$

With both solutions at $65^{\circ} \mathrm{C}$., the silver was run into the bromide solution in a fine stream with rapid mechanical stirring in $25 \pm 2$ minutes. Directly after this, $80 \mathrm{~g}$ of gelatin, previously swelled in cold water and drained, was added in small amounts; it was completely dissolved in 8 to 10 minutes and the emulsion was then chilled rapidly. It was usually allowed to set over night before shredding and washing; even with active gelatin the ripening during this period is negligible. After washing, it was digested, this time at $55^{\circ} \mathrm{C}$., and known bromide- and hydrogen-ion concentrations. At appropriate intervals, samples were taken out and test plates coated. Results with this formula, using deactivated gelatin and sensitization by allyl thiocarbamide added before digestion are recorded in figure 3 .

A modification of the formula, which will be known as C-2, may be used for higher speeds with somewhat lower contrast.

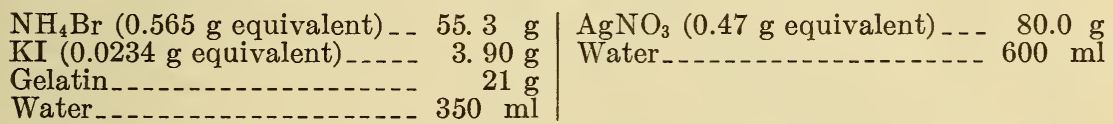

In this case, the AgI has been raised to 5 mol percent. The concentration of the bromide solution has been raised in order to maintain the solubility of the silver halide and thus the grain size. The solutions were mixed in $33 \pm 2$ minutes at $65^{\circ} \mathrm{C}$. After mixing, $110 \mathrm{~g}$ swelled gelatin was added in about 10 minutes and the emulsion chilled. Washing and digestion were carried out as for C-1.

One of the problems connected with any experiments on sensitizing compounds lies in the control emulsions. It is, as far as we know, impossible to prepare a gelatin which is truly inert photographically. Digestion after washing, at relatively low bromide-ion concentrations and $\mathrm{pH}$ of 6 or over, is apparently the most severe test of inertness; special gelatins stated by their manufacturer to be inert under other conditions were found to be quite active during digestion. As stated in a previous paper (12) some samples of gelatin can be very considerably deactivated by digestion with ammonia followed by thorough washing. About half the emulsions were made with a deactivated gelatin of this type which will be designated as "gelatin 1." "Gelatin 2 ", of about the same activity as the previous lot, was prepared from a batch of Nelson no. 2 gelatin. This was extracted first with dilute sulphuric acid, the $\mathrm{pH}$ of the gelatin being lowered to about 3 , then washed thoroughly in tap water, coming out approximately isoelectric. It was then extracted with dilute ammonia, and washed again; the final $\mathrm{pH}$ was about 7 . The control emulsions with these gelatins are recorded in figures 3 and 8. 


\section{SENSITOMETRY}

The experimental plates were tested by the standard methods of this bureau: Nonintermittent time-scale exposure at an intensity of 1 meter candle of sunlight quality, brush development in pyrogallol without bromide for 3,6 , and 12 minutes at $20^{\circ} \mathrm{C}$. and measurement of densities in diffuse illumination. The sector wheel usea in this laboratory is of the conventional Hurter and Driffield pattern with 9 steps in ratio of 2 . For all tests reported in this paper, 2 exposures were made, differing by 0.09 to 0.15 on the $\log E$ scale, so that 18 points on the characteristic curve were obtained. Use of the pyrogallol developer has been continued for comparison with earlier work. Some of the emulsions were also developed with the standard $p$-aminophenol formula (28) and with the metol-hydroquinone formula tentatively recommended by Davis and Neeland (29). As far as could be determined, the results are parallel with any of these developers; there is

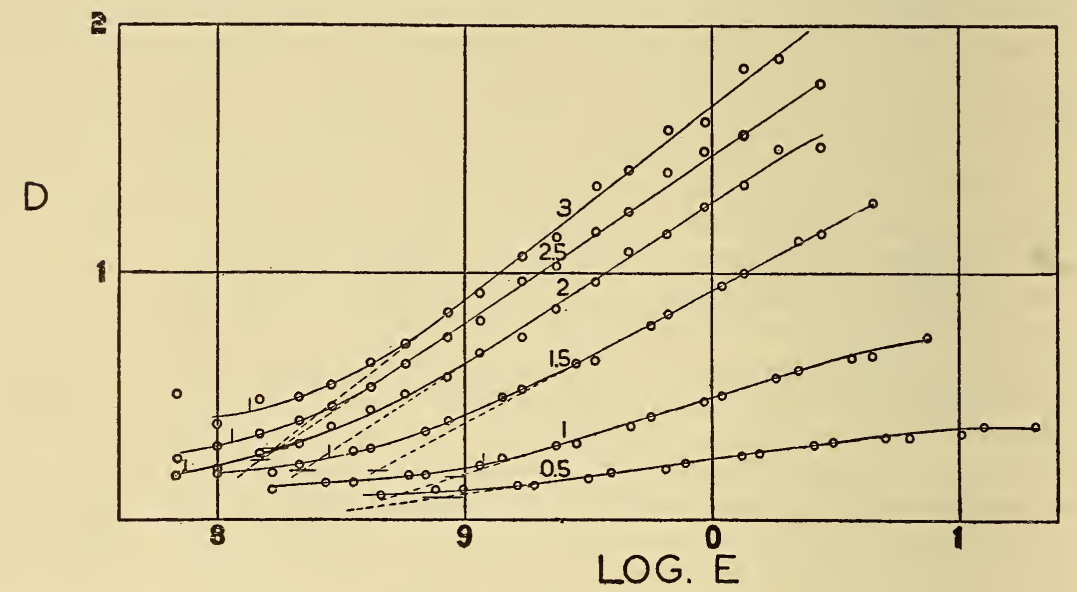

Figure 1.-Characieristic curves for 6-minuie development of emulsion 4-178 after digestion in vacuum with sodium sulphite for $1 \frac{1}{2}, 1,1 \frac{1}{2}, 2,21 / 2$, and 3 hours.

C-2 emulsion formula made with gelatin 2 , digested with $1.7 \mathrm{mg}$ sodium sulphite per $\mathrm{g}$ silver bromide at $55^{\circ}$, $\mathrm{pH} 7.4[\mathrm{Br}-] 5 \times 10^{-5}$. This amount of sulphite corresponds to a ratio of $6 \times 10^{-3}$ in terms of chemical equivalents.

no indication that the sulphite sensitization introduces any peculiarities of development.

Representation of the results without resorting to an undesirable use of tables gives the usual difficulties. The change in sensitivity on digestion with sulphite is such that the shape of the characteristic curve is altered, with changes in speed, contrast, and relative length of "toe", very much like the corresponding results on digestion of a normal emulsion (27). 'The type of change is illustrated by the characteristic curves plotted in figure 1 and the corresponding numerical values in table 1. After comparing different methods of plotting the results, it was decided to use the value of $10 / E_{m}$ (where $E_{m}^{\prime}$ is the exposure corresponding to the "minimum useful gradient" of 0.2 ) for 6-minute development as the best approximation to a single inde $x$ of sensitivity. The characteristic curves of either type of emulsion have satisfactory straight-line portions and it would be possible in 
most cases to give the data in the form of $10 / i$ at a constant $\gamma$. However, this would exclude a number of batches in the early stages of digestion, and would give little improvement in the accuracy of representation of the individual points. Our conclusions are, of course, in all cases based on comparison of the complete characteristic curves, and not merely on the numbers plotted in our figures.

TABLE 1.-Sensitometric data for emulsion 4-178 at varying time of digestion with sodium sulphite; cf. figure 1, characteristic curves at 6-minute development, and figure 6, speed $\left(10 / E_{m}\right)$ vs. digestion time

\begin{tabular}{|c|c|c|c|c|c|c|c|c|c|c|c|c|}
\hline \multirow{3}{*}{ Digestion time, hours } & \multicolumn{6}{|c|}{ Speed } & \multirow{2}{*}{\multicolumn{3}{|c|}{$\gamma$}} & \multirow{2}{*}{\multicolumn{3}{|c|}{ Fog }} \\
\hline & \multicolumn{3}{|c|}{$10 / E_{m}$} & \multicolumn{3}{|c|}{$10 / i$} & & & & & & \\
\hline & 3 & 6 & 12 & 3 & 6 & 12 & 3 & 6 & 12 & 3 & 6 & 12 \\
\hline $\begin{array}{l}0.5 \\
1.0 \\
1.5 \\
2.0 \\
2.5 \\
3.0\end{array}$ & $\begin{array}{r}87 \\
175 \\
320 \\
600 \\
460\end{array}$ & $\begin{array}{r}87 \\
400 \\
1,300 \\
1,300 \\
1,000\end{array}$ & $\begin{array}{r}25 \\
150 \\
590 \\
1,250 \\
1,380 \\
1,400\end{array}$ & $\begin{array}{r}87 \\
170 \\
310 \\
470 \\
740 \\
560\end{array}$ & $\begin{array}{l}100 \\
220 \\
345 \\
590 \\
790 \\
580\end{array}$ & $\begin{array}{r}72 \\
140 \\
325 \\
525 \\
760 \\
490\end{array}$ & $\begin{array}{l}0.12 \\
.24 \\
.37 \\
.44 \\
.42 \\
.50\end{array}$ & $\begin{array}{r}0.15 \\
.30 \\
.53 \\
.64 \\
.67 \\
.82\end{array}$ & $\begin{array}{r}0.27 \\
.65 \\
.77 \\
.95 \\
1.00 \\
1.26\end{array}$ & $\begin{array}{r}0.00 \\
.02 \\
.03 \\
.05 \\
.08 \\
.12\end{array}$ & $\begin{array}{l}0.02 \\
.03 \\
.05 \\
.07 \\
.13 \\
.21\end{array}$ & $\begin{array}{r}0.08 \\
.11 \\
.13 \\
.13 \\
.18 \\
.29\end{array}$ \\
\hline
\end{tabular}

\section{SPECTRAL SENSITIVITY OF SULPHITE-SENSITIZED EMULSIONS}

No change in relative spectral sensitivity on sensitizing with sulphite has been evident in any case, but in view of the nature of the sensitizing produced by bathing in bisulphite solutions (the Capstaff effect) it was advisable to make a careful determination of this feature of the process. The effect to be anticipated is an extension of the natural sensitivity to the longer wave lengths. In order to detect this, exposures were made with a glass prism spectograph using a gas-filled incandescent lamp as light source. The instrument which was used is quite free from stray light, but in order to reduce both this difficulty and the spreading of the image by scattering of light within the emulsion itself, a Wratten "minus blue" filter was placed over the slit. ${ }^{2}$ The plates were brush developed to insure uniformity. The plates which were compared were taken from two enulsions made by the C-2 formula under conditions which were identical within the limits of control. Having 5 percent AgI, the sensitivity extended into the longer wave lengths relatively far for emulsions containing no dye (30). Figure 2 gives curves derived from exposures of a control (unsensitized) plate and two plates sensitized with sulphite and allyl thiocarbamide, respectively. All three were developed to a $\gamma$ of 0.50 . Similar results were obtained in other experiments.

It is evident that, by sufficiently accurate choice of exposure, it would be possible to have close coincidence of the density-wave length curves for the batches sensitized with sulphite and thiocarbanide, but that the control emulsion shows a distinctly more abrupt decrease of density with increasing wave length. This indicates that either type of sensitivity nucleus causes a very slight extension of spectral sensitivity. Marked panchronatic sensitization such as the Capstaff effect is obviously not characteristic of sensitization by sulphite under all conditions. As all our experimental emul-

This particular filter had a transmission of 12.5 percent at $5,000 \mathrm{~A}$ and 1.5 percent at 4,900 . 
sions have been given a preliminary test exposure behind an EderHecht wedge with its filter strips, any marked spectral sensitization would have been detected; none occurred.

Limited data are available on another question involving spectral sensitivity - the interrelation of sensitization by sulphite and by dyes. Sulphite, when added to the emulsion just before coating, may (31) have an appreciable hypersensitizing effect on the dye, the sensitivity to the longer wave lengths being selectively increased without a corresponding increase in the sensitivity to the blue. This is, however, distinct from the case of an emulsion which has been digested with sulphite so as to produce an increase in sensitivity independent of the action of dyes, then made color sensitive by further treatment with a dye. In such cases, the relative spectral sensitivity conferred by the dye appeared to be normal. In the most complete test of this feature of sulphite sensitization, sulphite was added to the emulsion after washing in the ratio of $2.5 \mathrm{~g}$ equivalents to 1,000 of silver bromide. Samples were coated directly after this and after two periods

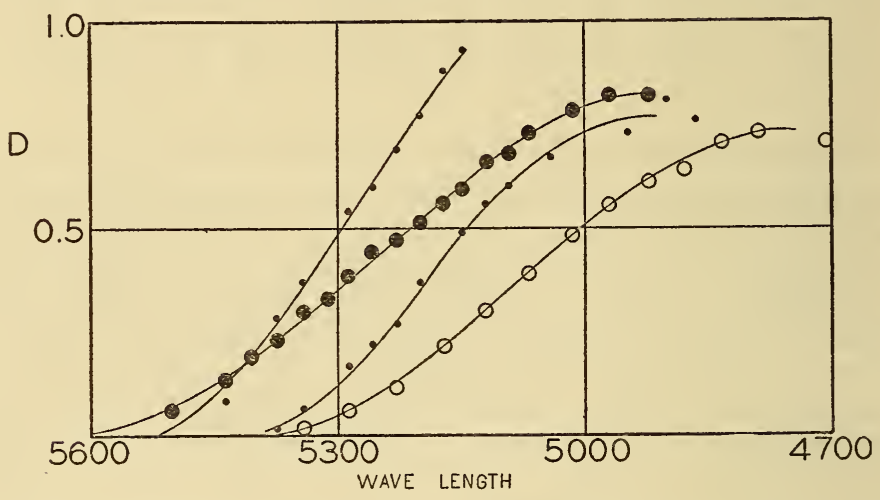

FIgURE 2.-Densities of plates made by $C$-2 formula, exposed in prism spectrograph through minus blue filter, and developed to gamma of 0.5 .

- = Unsensitized control, $=$ sulphite-sensitized batch, $\mathrm{O}=$ allyl thiocarbamide-sensitized batch. General fog density subtracted from all image densities

of digestion, each was sensitized with the same amount of pinacyanol. The resulting plates were tested for sensitivity to red and blue light by sensitometer exposures through Wratten $\mathrm{A}$ and $\mathrm{C}$ filters. Comparing the resulting speed numbers, ${ }^{3}$ the ratio $\mathrm{A} / \mathrm{C}$ was $3.1,4.7$, and 4.7 for the samples digested 0,1 , and 2 hours, respectively; the values for the undigested sample are the least reliable because of its low contrast. As the ratio remained approximately constant for a change in total sensitivity of 8 to 1 , the evidence is that the formation of silver nuclei by sulphite sensitization is quite analogous to the action of allyl thiocarbamide in its effect on sensitization by dyes (6) (32).

The sulphite might have been expected to interfere with sensitization by reducing and decolorizing the dye. Apparently the dye is protected by its adsorption on the silver bromide, since sulphite in slightly larger quantities may even have an appreciable hypersensitizing action (31, table 8). The emulsion ripened with sulphite and

3 These values are arbitrary, since they are not corrected for energy distribution of the source and transmission of the filters. Comparison was made at the constant development time of 6 minutes for each plate, since the digested and undigested samples could not be compared at the same value of gamma. 
sensitized with pinacyanol, and that to which sulphite was added just before coating for the tests of hypersensitizing action, were tested again (with the Eder-Hecht wedge) after 9 months' storage. There was no evident change in relative spectral sensitivity in any case, indicating that the sulphite had not reduced the dye on storage. The white-light sensitivity had increased or decreased in some cases, depending on the degree of ripening of the emulsion.

\section{THE EFFECT OF CONCENTRATION OF SENSITIZER}

Other conditions being constant, the rate of after-ripening would be expected to increase continuously with the sulphite concentration, and we have found this to be the case within the limits of concentra-

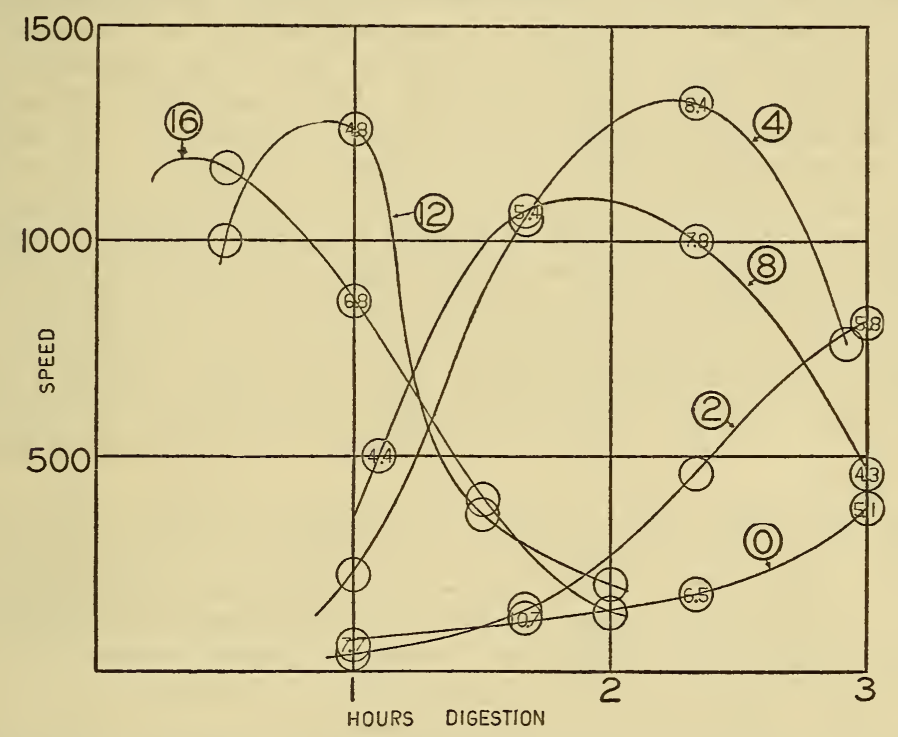

FIGURE 3.-Emulsions made by C-1 formula, gelatin 1, and sensitized by digestion with allyl thiocarbamide after washing.

The numbers of the curves indicate the ratio of allyl thiocarbamide to silver bromide in terms of chemical equivalents; for example, the figure 2 means that 2 atoms of silver per 100,000 could be converted from silver bromide to silver sulphide by the allyl thiocarbamide. Figures inside the circles representing experimental points give the nonhalide silver found in the emulsion by analysis after the digestion indicated by the location of the point; these numbers are also to be divided by $10^{5}$ to give the ratio of the nonhalide silver to silver bromide in terms of chemical equivalents.

tion which were tested. The sensitivity, however, passes through a maximum on continued after-ripening under any conditions, and the value of this maximum is not necessarily independent of sensitizer concentration. Sheppard (33) reported that the sensitivity passed through a maximum with increasing concentration of allyl thiocarbamide, the decrease in speed being much more than would be expected simply from the increase in fog. As the conditions of his experiments were not described, we present in figure 3 the results of a similar series under the same conditions as the experiments with sulphite shown in figure 4 (C-1 emulsion formula, gelatin 1, sensitizer added after washing) for comparison with the latter. The curves of figure 3 show a progressive increase in rate of after-ripening from the control to the batch with the largest sensitizer concentration. The maxi- 
mum value of each curve is not much different, on this basis of representation, for the concentrations from 4 to $16 \times 10^{-5} \mathrm{~g}$ equivalent of thiocarbamide per $\mathrm{g}$ equivalent of $\mathrm{AgBr}$; the curve for $2 \times 10^{-5}$ does not reach its maximum, but the value would evidently be lower. The apparent results are in this case unfortunately affected by the method of representation; using $10 / i$ at a constant value of $\gamma$ for the speed number instead of $10 / E_{m}$, the maximum speed was obtained with a ratio of $8 \times 10^{-5}$ and 1 hour 40 minutes digestion; the maximum for $4 \times 10^{-5}$ was decreased with respect to the others, while that for $2 \times 10^{-5}$ was increased. These data indicate that the sensitivity obtainable from a given emulsion is relatively independent of the amount of sensitizer over a moderate range; this in agreement with the general experience of emulsion makers that the potential sensitivity of an emulsion is largely determined by the conditions of emulsification - the early stages of the making where the composition and size-distribution of the grain are fixed. Obviously the range of sensitizer concentration for which this holds is limited. The amounts used in these experiments are in the optimum region for these conditions, but are well below the optimum ratio calculated from Sheppard's (33) figures. It is desirable to repeat that this relatively constant maximum sensitivity can be secured only by proper adjustment of digestion conditions. For constant digestion, the sensitivity passes through a sharp maximum with changing amount of sensitizer. For example, at 1 hour the maximum sensitivity was obtained with a ratio of $12 \times 10^{-5}$; at 2 hours the optimum ratio had changed to 4 or $8 \times 10^{-5}$ depending on the speed number used, and at 3 hours it was $2 \times 10^{-5}$.

It has been generally assumed in the literature that the reaction between allyl thiocarbamide and silver bromide was complete during digestion. The nature of the curves for speed against digestion time makes it evident that the reaction was nowhere near complete at maximum sensitivity. This was, therefore, tested chemically, using the modified Weigert and Lühr method (11) of analysis for nonhalide silver. The results are given by the numbers in the circles locating the points of figure 3 . Not all the plates were analyzed; those chosen correspond to the optimum digestion time for a given ratio (choosing the time giving the maximum 10/i), plus all times for the control and $8 \times 10^{-5}$ ratio. Analysis for these minute traces of material is subject to considerable error in the final determination, and still more in the separation of silver halide and nonhalide silver. The reliability has been tested on synthetic mixtures, however, with satisfactory results. The data indicate that the maximum sensitivity corresponded to the formation of nonhalide silver in amounts of the order of $5-8 \times 10^{-5} \mathrm{~g}$ equivalent per g equivalent of silver bromide, for all amounts of thiocarbamide. It is impossible to say how much of the nonhalide silver was silver sulphide and how much was metal. The values for the controls were disconcertingly high; similar results have been obtained with other insensitive emulsions (27). It seems probable that some of the nonhalide silver comes from the traces of colloidal silver present in the best commercial silver nitrate, or is formed during mixing from contact of the silver-nitrate solution with the gelatin, or both. This does not explain the existence of larger quantities in the control than in the batches to which thiocarbamide had been added. There are no reasons for suspecting these particular data, and they are given 
for what they are worth. The decrease in nonhalide silver on continued digestion has been observed before (27); oxidation of metallic silver or silver sulphide of colloidal dimensions is not improbable under the conditions of digestion, and would lead to a decrease in the silver remaining after fixation.

The data presented in figures 4,5 , and 6 show the expected increase in rate of after-ripening with increase in sulphite. For the C-1 emulsions, figure 4 , the time required to reach a given sensitivity is, very roughly, inversely proportional to the sulphite- $\mathrm{AgBr}$ ratio. For the C-2 emulsions, figures 5 and 6 , the change in rate of afterripening is much less than the change in sulphite. It will be noted at once that the quantities of sulphite used are of the order of 20 to 50 times as great as the allyl thiocarbamide used for the same effect

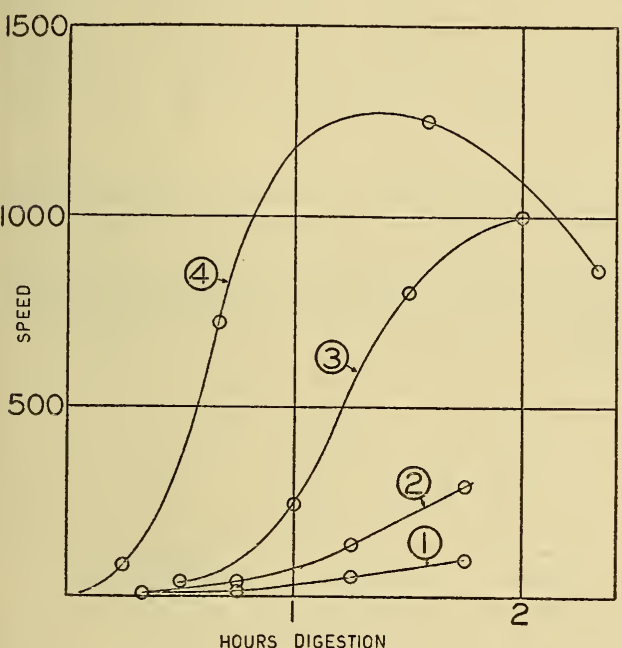

FIGURE 4-Emulsions made by the $C-1$ formula, gelatin 1 , and sensitized by digestion with sodium sulphite after washing, in vacuum at $55^{\circ}$, $\mathrm{pH} 6.8\left[\mathrm{Br}^{-}\right] 3-5 \times 10^{-5}$.

Ratio of sodium sulphite to silver bromide in terms of chemical equivalents. Curve 1,0 ; curve $2,5 \times 10^{-4}$; curve $3,1.3 \times 10^{-3}$; curve $4,2.4 \times 10^{-3}$. That is, the sulphite added to the emulsion giving curve 4 was sufficient to reduce 2.4 atoms of silver per 1,000 from silver bromide to metallic silver.

most (assuming that the nonhalide silver in the undigested portione 列 was there before addition of the sulphite). If this is correct, silver is equivalent to a larger amount of silver sulphide ${ }^{4}$ rather than a lesser one. For either sensitizer the optimum amount of nonhalide silver present as sensitivity nuclei in our emulsions is about 1 to $5 \times 10^{-5}$, expressed in terms of its atomic ratio to silver halide. This is toward the lower limit of Sheppard's estimate (14).

The sensitivity of the emulsion passes through a maximum on continued digestion with sulphite, as in other methods of after-ripening. The height of the maximum is roughly constant over a moderate range

4 The nonhalide silver in the emulsions made with deactivated gelatin and sensitized with either allythiocarbamide or sodium sulphite was less than in emulsions made with active gelatin (27). 


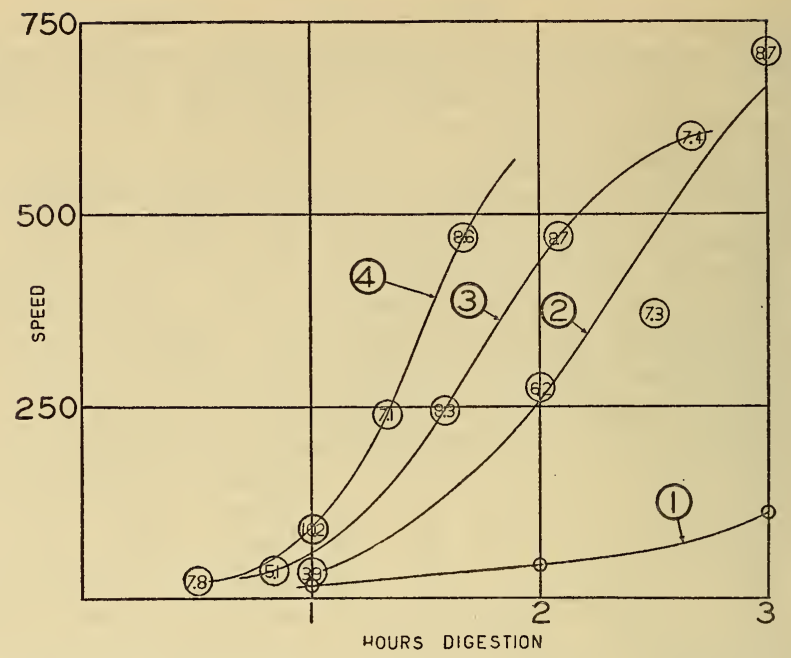

FIGURE 5.-Emulsion made by the C-2 formula, gelatin 2, and sensitized by digestion with sodium sulphite after washing, in beakers at $55^{\circ}, \mathrm{pH} 6.7\left[\mathrm{Br}^{-}\right] 1.3 \times 10^{-5}$.

Curve 1, control emulsion digested under same conditions without sulphite. Ratio of sulphite to silver bromide: Curve 2, $1.9 \times 10^{-3}$; curve 3, 3.8 $\times 10^{-3}$; curve 4, 7.6 $\times 10^{-3}$. Numbers in the circles locating experimental points give the nonhalide silver found in the emulsion by analysis after the digestion indicated by the location of the point; these numbers are to be divided by $10^{5}$ to give the ratio of the nonhalide silver to silver bromide in terms of chemical equivalents.

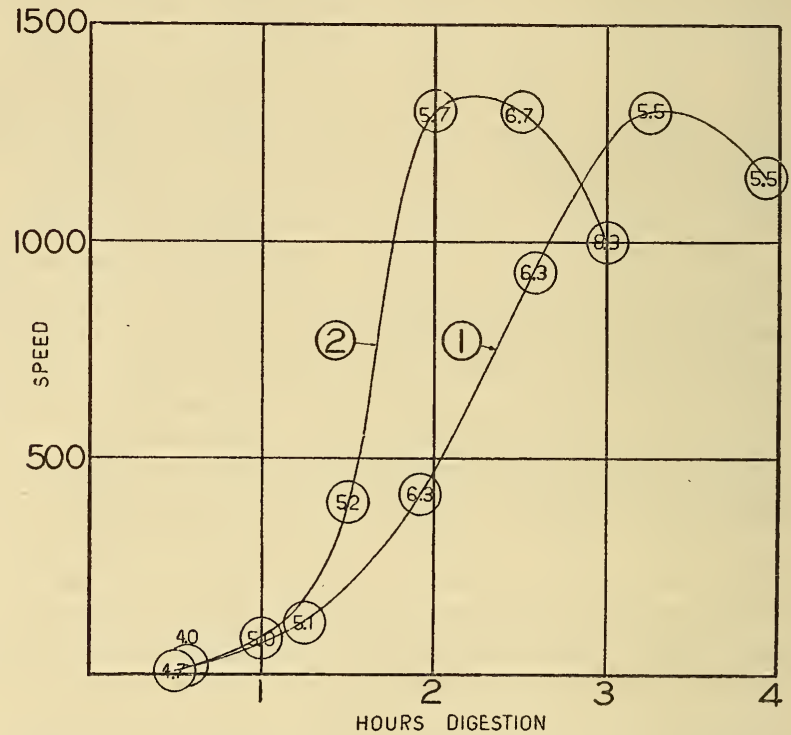

FIguRE 6.-Emulsion made by the C-2 formula, gelatin 2, and sensitized by digestion with sodium sulphite after washing, in vacuum at $55^{\circ}, \mathrm{pH} \% .4[\mathrm{Br}-] 5 \times 10^{-5}$.

Ratio of sulphite to silver bromide: Curve 1, $1.5 \times 10^{-3}$; curve 2, $6 \times 10^{-3}$. Numbers in the circles locating experimental points give the nonhalide silver found by analysis in the emulsion after the digestion indicated by the location of the point; these numbers are to be divided by $10^{5}$ to give the ratio of the nonhalide silver to silver bromide in terms of chemical equivalents. 
of conditions; ratios of sulphite to silver bromide of $1.5 \times 10^{-3}$ or over appeared to differ mainly in the rate of change (figures 6 and 10 show well-defined maxima with different concentrations). Using the same formula, the maximum obtainable with sulphite was slightly lower than that with allyl thiocarbamide; the difference in the numerical values used for plotting the curves was very small, but it was larger on the basis of $10 / i$. The results with these two essentially different sensitizers are still close enough to emphasize that the maximum sensitivity is determined more by the composition, structure, and size of the grains than by the subsequent ripening processes.

Results with the $\mathrm{C}-1$ and $\mathrm{C}-2$ formulas are parallel. A number of experiments were made with emulsions of pure silver bromide or with 1 percent AgI. Such emulsions have less capacity for sensitization, as judged from experience with after-ripening under normal conditions (27) and from the literature on emulsions. It is necessary to adjust the time and temperature of mixing in order to secure the same grain size and distribution in emulsions with varying iodide content (27), since the increased solubility of the silver halide with decreasing iodide results in an increase in grain size if the formula is otherwise unchanged. This was worked out quite well for the emulsions with 1 percent AgI; the few emulsions made with pure bromide had a coarser grain. The results proved difficult to reproduce and the experiments were not completed, but it was evident that under the same conditions the sensitization of these emulsions by sulphite was much less than for those with 4 to 5 percent AgI, and that the purebromide emulsions were the worse in this respect. Our earliest experiments with sulphite happened to be with pure-bromide emulsions made by the ammonia process. In these, increases in speed of 50 percent over the control were obtained; comparative experiments with allyl thiocarbamide gave the same results.

Oxidation of the sulphite by air during digestion is a complication of the ripening process. Our emulsions are normally digested in open beakers with stirring to prevent settling; the exposed surface is large enough so that the liquid may be expected to be saturated with air. The effect of the aerial oxidation is apparently less than would be expected, most probably because adsorption of the sulphite to silver bromide protects it. Comparative results of digestion with and without exclusion of air are given in figure 7. Digestion in vacuum was accomplished by pouring the freshly melted emulsion into a balloon flask provided with a special low-sulphur stopper ${ }^{5}$ through which was run a stopcock. The flask was evacuated repeatedly, usually with small additions of alcohol, until the froth was broken and the warmed emulsion boiled vigorously under the reduced pressure. The sulphite solution was then introduced through the stopcock without breaking the vacuum. The samples for coating were removed by inverting the flask over a bottle with a 2-hole stopper, through which the stopcock passed tightly, evacuating the bottle and opening the stopcock when the pressure was below the vapor pressure in the flask. The flask was shaken violently before taking a sample, or every half hour if the sampling was less frequent; settling was negligible. Comparing curve 1 with curve 2, and curve 3 with curve

$5 \mathrm{We}$ are indebted to the rubber section of this bureau for making these stoppers; comparison of control batches digested in flasks fitted with them with those digested in the usual way demonstrated that they did not cause fog or sensitization. 
4 , the indications are that the loss of sulphite by oxidation was relatively small. The rate of after-ripening in vacuum was definitely faster in curve 4 than in curve 3 ; in this emulsion $4-127$, the concentration of sulphite was less than in 4-124, curves 1 and 2 , so that the effect of oxidation might be larger. Curves 1 and 2 might legitimately be drawn in practical coincidence, although the form chosen for curve 2 is the most probable considering its points alone. Later experiments were generally made in vacuum, although in some cases, which are indicated, the open beakers were used for convenience. It was later decided to make experiments with more careful exclusion of oxygen. In these, the same apparatus was used as for the vacuum digestion, but the flask was repeatedly evacuated and flushed out with nitrogen or hydrogen. Digestion under hydrogen was found to

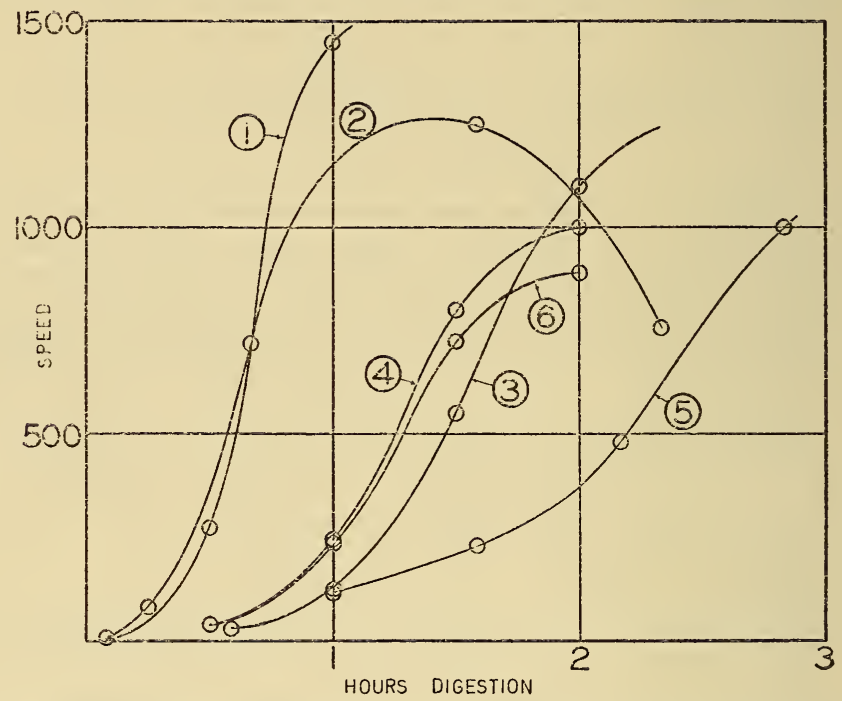

FIGURE 7.-Comparison of sensitization of emulsions digested with sulphite after washing with and without free access of air.

Curves 1 and 2, emulsion 4-124, C-1 formula, gelatin 1; digested at $55^{\circ} \mathrm{pH} 6.8$ [Br-] $5.6 \times 10^{-5}$, sulphitesilver bromide ratio $2.4 \times 10^{-3}$; curve 1 in beaker, curve 2 in evacuated flask. Curves 3 and 4 , emulsion 4-127, $\mathrm{C}-1$ formula, gelatin 1 ; digested at $55^{\circ}, \mathrm{pH} 6.7,\left[\mathrm{Br}^{-}\right] 2.9 \times 10^{-5}$, sulphite-silver bromide ratio $1.3 \times 10^{-3}$; curve 3 in beaker, curve 4 in evacuated flask. Curve 5, emulsion 4-180, C-1 formula, gelatin 2; digested at $55^{\circ}$, pH 6.5 [Br- $2.6 \times 10^{-5}$, sulphite-silver bromide ratio $1.5 \times 10^{-3}$ in evacuated flask. Curve 6, emuision 4-186; conditions identical with $4-180$ except that flask was filled with nitrogen.

cause heavy fog, accompanied by an increased rate of after-ripening in the early stages of the process. It was at first thought that this indicated extensive oxidation of the sulphite even in the vacuum digestion, but direct comparison with nitrogen proved that the fog came from the hydrogen. ${ }^{6}$ It is possible that the colloidal silver formed by the sulphite catalyzed further reduction of the silver bromide by the hydrogen. Curves 5 and 6 compare the digestion of C-2 emulsions in vacuum and nitrogen; the other halves of these emulsions were digested under hydrogen. The results indicate a considerable oxidation of the sulphite in the vacuum run, but are less reliable because curves 5 and 6 are for different emulsions.

\footnotetext{
${ }_{6}^{6}$ Both gases were freed from oxygen by passing slowly through a spiral wash bottle filled with fresh
} alkaline pyrogallol. 
Fog densities corresponding to the maximum sensitivities shown in the figures were from 0.06 to 0.13 corrected for the density of the support. The fog densities of emulsions sensitized with allyl thiocarbamide to the same range of sensitivity fell within the same range; using active gelatins, the values were about the same for the best samples. It is a distinct practical advantage for the sulphite that the effect of concentration is not so critical as with other sensitizers. For example, the longest time of digestion of emulsion 4-178 with the higher concentration, $6 \times 10^{-3}$, was $1 / 3$ more than the optimum, and the decrease in sensitivity was about $1 / 4$; the corresponding fog was only 0.21 . Under corresponding conditions with thiocarbamide or active gelatin, the fog has usually been 0.3 to 0.4 .

Adding the amounts necessary to give a reasonable digestion time, only a small percentage of the sulphite is utilized, so that it was feared that the plates would fog rapidly on storage. This has not been the case; the stability has been rather better than normal on the plates which have been tested. The tests were made after 1 year storage, repeating the Eder-Hecht wedge exposure used for the preliminary test, with tank development in pyro giving fog about the same as 6-minute brush development. Fog densities after 1 year for the plates corresponding to the maximum speeds on the curves are as follows: Figure 4, curve 3, fog 0.21 ; figure 4, curve 4, fog 0.18 ; figure 7 , curve 1 , fog 0.14 ; figure 10 , curve 4 , fog 0.27 . Speed was in all cases constant as closely as could be determined by Eder-Hecht exposures. These are satisfactory values after 1 year in the climate of Washington. In all 4 cases cited, the emulsion had been given a thorough washing, digested, and coated without addition of soluble bromide, the bromide-ion concentration being 2 to $3 \times 10^{-5} \mathrm{~N}$. For practical use, bromide would have been added after digestion in the ratio of about $3 \mathrm{KBr}: 1,000 \mathrm{AgBr}$, raising the bromide-ion concentration to 5 to $8 \times 10^{-4} \mathrm{~N}$; this would avoid the edge fog which was obvious for a few $\mathrm{mm}$ from uncut edges of the plates, as well as increasing the time of stability. The unexpectedly high stability is probably explained by oxidation of the sulphite by air during drying which eliminates the excess sensitizer. Analytical data in proof of this have not been obtained, as time was not available to attempt a test of such obvious difficulty.

\section{HYDROGEN-ION CONCENTRATION}

The rate of after-ripening with sulphite is less sensitive to hydrogenion concentration than the corresponding process in active gelatin; the data in figure 8 illustrating the variation with $\mathrm{pH}$ may be compared with the corresponding figures 2 and 3 of B.S. Research Paper No. 430. Control batches for each value of $\mathrm{pH}$ have been included in figure 8 , as the activity of treated gelatins has been found to be liable to be highly dependent on this variable. The data on sulphite sensitization which are illustrated were obtained by vacuum digestion. Two sets of experiments by digestion in beakers gave results less dependent on $\mathrm{pH}$ and falling in no regular order, although in both cases the batch at 6.7 gave a minimum rate. The most probable 
explanation of this behavior lies in competition for the sulphite between the silver bromide and the oxygen of the air; oxidation by the air being highly dependent on hydrogen-ion concentration, the reaction between silver bromide and sulphite might actually assume a minimum at some $\mathrm{pH}$. Our data show no indication of systematic

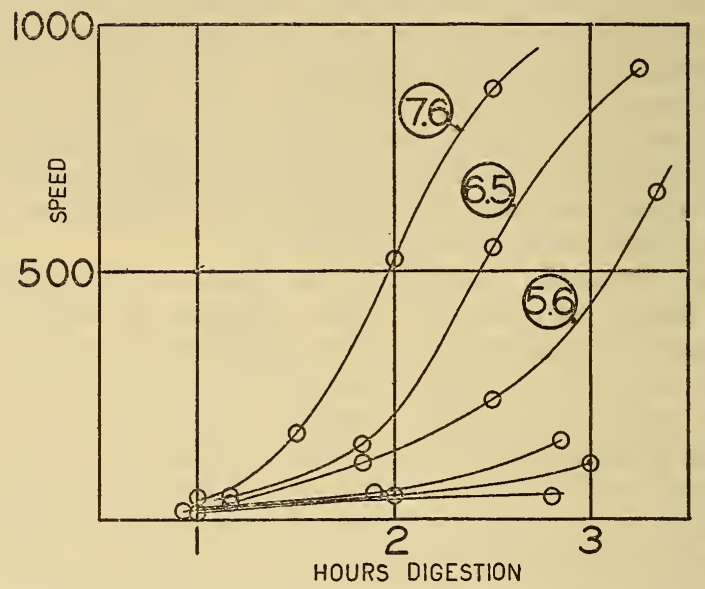

FiguRe 8.-Comparison of sensitization of emulsion digested with sodium sulphite in vacuum after washing, at varying $\mathrm{pH}$.

Emulsion 4-177, C-2 formula, gelatin 2; [Br-] as washed $4.6 \times 10^{-5}$. Numbers on the curves indicate $\mathrm{pH}$ of digestion. The lower curves are for digestion of a control emulsion without sulphite at the same values of $\mathrm{pH}$.

difference in the maximum sensitivity obtainable at different hydrogen-ion concentrations within the range tested.

\section{BROMIDE-ION CONCENTRATION}

After-ripening with active gelatin or with pure sensitizers such as allyl thiocarbamide is retarded by increase in bromide-ion concentration, but the sensitivity reaches its maximum value by digestion at a bromide-ion concentration which may be as high as $1 \times 10^{-3} N(27)$. Since bromide ions are also a product of the ripening with sulphite, similar relations may be expected to hold. Data obtained with C-2 emulsions are presented in figure 9, those from C-1 emulsions in figure 10. The reaction rate is more sensitive to bromide-ion concentration than to hydrogen-ion concentration, but the factor is less than 2 as far as it can be estimated from the time required to reach a given sensitivity. Neither approaches the effect of the concentration of sulphite; the time for a given effect was not more than doubled when the bromide-ion concentration was multiplied by 10 . The effect of bromide-ion concentration on the sulphite sensitization is at least as large as on the corresponding process with allyl thiocarbamide, and is much greater than when sodium thiosulphate or active gelatin is used (27). Contrary to the case of normal after-ripening (with active gelatin), the maximum sensitivity is not improved by addition of soluble bromide to a thoroughly washed emulsion before digestion; with sulphite the more rapid after-ripening led to results which were always as good and in some cases better. This may possibly be 
explained by the oxidation of sulphite by air from leakage into the flask with consequent serious loss before the end of a long digestion, but it seems more likely to be characteristic of the process. As bromide may be added after digestion as a preservative, it is obviously

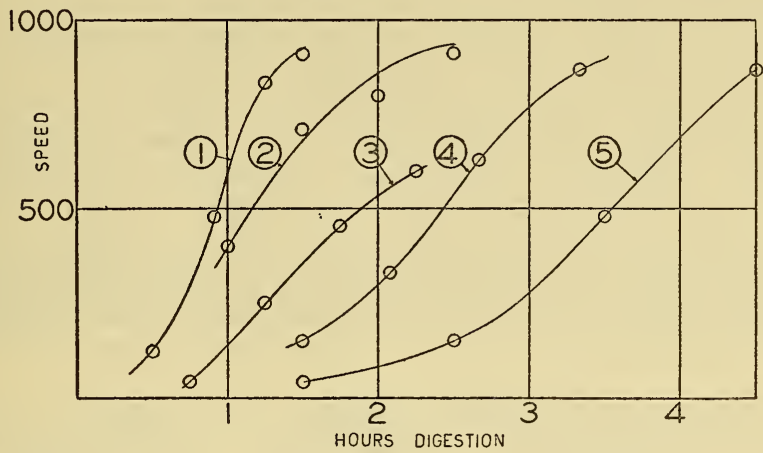

FIGURE 9.-Comparison of sensitization of emulsions digested with sodium sulphite after washing in vacuum at varying bromide-ion concentrations.

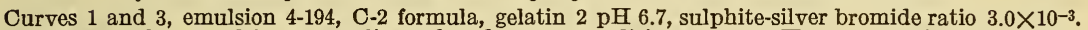
curves 2, 4, and 5, emulsion 4-179; digested under same conditions except $\mathrm{pH}$ 7.7. Bromide-ion concentrations [Br-]: Curve 1, $3.1 \times 10^{-6}$; curve 2, $1.0 \times 10^{-5}$; curve $3,2.6 \times 10^{-5}$; curve $4,1.2 \times 10^{-4}$; curve 5 , $4.8 \times 10^{-4}$.

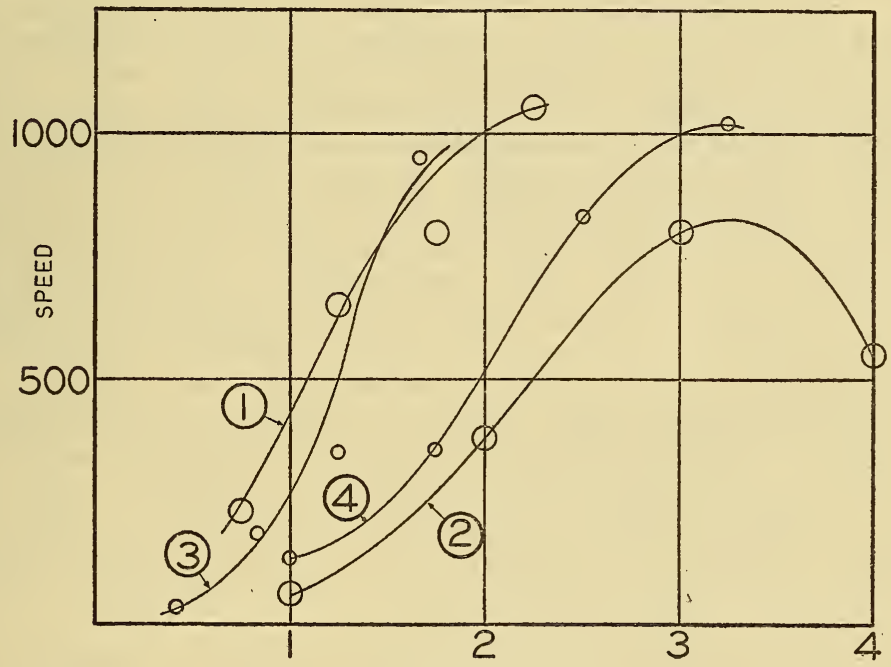

HOURS DIGESTION

Figure 10.-Comparison of sensitization of emulsions digested with sodium sulphite after washing at varying bromide-ion concentrations.

Emulsion 4-140, C-1 formula, gelatin 1; digested in beakers at $55^{\circ}, \mathrm{pH}$ 6.6, sulphite-silver bromide ratio $3 \times 10^{-3}$; curve $1,[\mathrm{Br}-] 3.1 \times 10^{-5}$, curve $2,9.4 \times 10^{-4}$ Emulsion $4-134$, C-1 formula, gelatin 1 ; digested in evacu
ated flasks at $55^{\circ}, \mathrm{pH} 6.5$, sulphite-silver bromide ratio $1.3 \times 10^{-3}$; curve 3 [Br-] $1.5 \times 10^{-6}$, curve $4,2.4 \times 10^{-5}$.

better practice to digest the emulsion after thorough washing $\left(\left[\mathrm{Br}^{-}\right]\right.$ around $2 \times 10^{-5}$ ) and add the preservative before coating.

\section{EMULSIONS WITH SULPHITE ADDED BEFORE WASHING}

Most of the experiments were made by the additions of sulphite to the washed emulsion, since this permitted control of the sulphite concentration and made the observation of its effect simpler than when 
change in grain size or other processes may be taking place. For practical use, it might be preferred to add the sulphite to the emulsion before washing (although on washing with hard water there might be difficulty with precipitation of calcium sulphite). There would then be the alternatives that the silver nuclei might be formed during the ripening process before washing, or that adsorbed sulphite (like adsorbed thiocarbamide) might be retained through the washing and function during subsequent digestion. There is the further possibility, independent of these, that the sulphite may affect the grain size if present before washing. Its solvent action on silver bromide is sufficient so that it could accelerate the growth of grains. However, in the presence of soluble bromide there will be a mutual reduction in the action of these two solvents caused by competition to form the corresponding complex silver ions, so that under some conditions the addition of sulphite might retard the ripening as observed by Valenta (18).

In the presence of ammonia, reduction by sulphite is relatively rapid and it is reported (13) to cause fog. While conditions might be adjusted to avoid this, the limited number of experiments which we were able to make were all with neutral emulsions, appropriately modified from the type formulas given on page 747. In all but one case, the sulphite was added to the bromide solution before mixing. Photomicrographs of the grain of these emulsions were compared with those of other emulsions in which conditions were the same except for the presence of the sulphite; there was no evident difference in grain size or size distribution. In the one exception (emulsion 4-138, table 2), the sulphite was added directly after completing the mix and the emulsion digested with it for an hour at $65^{\circ}$ before washing. Photomicrographs of the grain before and after this process showed little change in grain size, apparently less than from digestion with the same concentration of soluble bromide without the sulphite. The evidence from the characteristic curvecontrast, scale, and underexposure region-confirms that from the photomicrographs, since these characteristics are practically the same as in emulsions mixed without the sulphite and sensitized by other means. It had been expected that when sulphite was present in quantity during mixing, the increase in solubility of the silver bromide would decrease the contrast. The time of mixing was accordingly reduced for emulsion 4-134, but the contrast both for this and for 4-136 was very nearly the same as for emulsions made by the same formula without the sulphite.

There was no indication of reduction of the silver halide by the sulphite before washing in any case; portions of the emulsion coated without digestion were invariably of low sensitivity, practically indistinguishable from controls. In the case of emulsions 4-189 and 4-190, the excess of bromide over silver had been reduced so that the bromide-ion concentration was only $5.6 \times 10^{-3} \mathrm{~N}$ after adding all the silver solution. During addition of the secondary gelatin before chilling, the emulsions were held at $65^{\circ} \mathrm{C}$., $\mathrm{pH} 7.3$ and the above bromide-ion concentration for 10 minutes, but the undigested emulsions were as insensitive as others with more bromide and less sulphite. It is probable that continued digestion under these conditions would have produced some sensitization. While we were unable to test this, there is little reason to expect that the results 
would be superior to those which we obtained by digestion of the emulsions after washing. In this case, the after-ripening is best explained by adsorbed sulphite left after washing. A less probable hypothesis, concerning which we have no evidence, is that some new sensitizing compound has been formed by digestion of the sulphite with the deactivated gelatin during mixing of the emulsion. The after-ripening was of the normal type, the results being very similar to those where sulphite was added to the emulsion after washing. The nature of the data is such that they must be tabulated, and for economy of space we present only the optimum results for each digestion.

TABLE 2.-Emulsions with sodium sulphite added to bromide solution before mixing. Volumes of solutions as for $C-1$ or $C-2$ formulas. Mixed in 26-29 minutes at $65^{\circ}$ except as otherwise stated; digested after washing at $55^{\circ}, \mathrm{pH} 7.1 \pm 0.3,\left[\mathrm{Br}^{-}\right]$ $2.5 \pm 1 \times 10^{-5}$

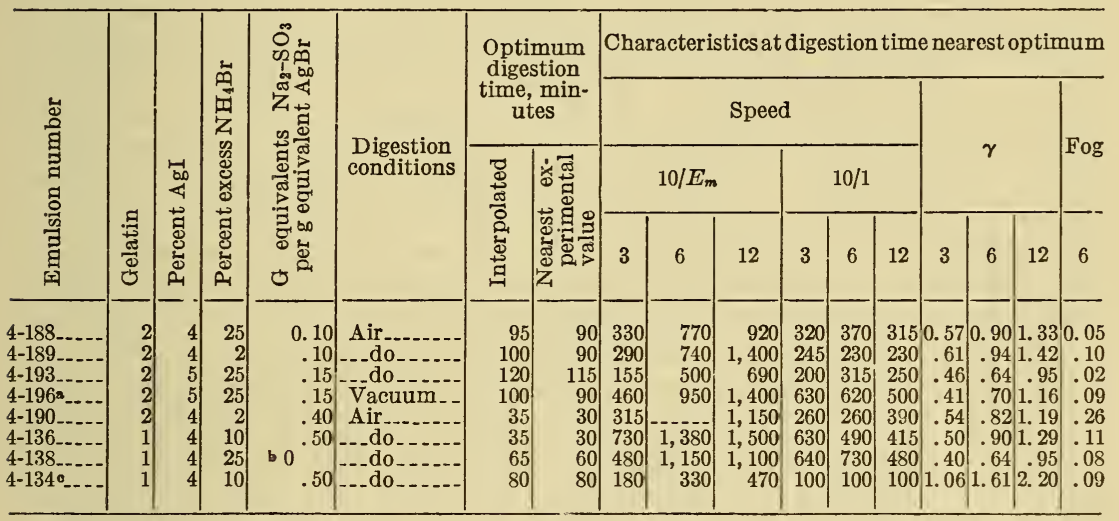

a Mixed at $70^{\circ} \mathrm{C}$. instead of $65^{\circ}$.

- No sulphite in mix; directly after mix added $\mathrm{Na}_{2} \mathrm{SO}_{3}$ in $0.50: 1$ ratio and digested 1 hour at $65^{\circ}$ before adding secondary gelatin and chilling.

- Mixed in 14 instead of 26 minutes.

As would be expected, the rate of after-ripening increased with increasing amounts of sulphite (compare 189 with 190 , and 188 with 136). The emulsions in which the excess bromide in the original mix had been reduced to its lowest value (189 and 190) had appreciably more fog than those with normal excess bromide; an increase in adsorption of the sulphite under these conditions is theoretically possible, but there is no evidence for it in the form of increased rate of after-ripening. The 2 emulsions with 5 percent $\mathrm{AgI}$ after-ripened slower than those with 4 percent, as is to be expected. Indications from the results with emulsions 4-193 and 4-196 are that still faster emulsions might have been obtained by increasing the amount of sulphite in the same basic formula.

Results with emulsions of this type were quite similar to those when the sulphite was added after washing. This process might be preferred for practical use. As already stated, the digestion could probably be eliminated by using a small excess of soluble bromide in the original solutions and ripening after mixing, but this is less likely to be satisfactory than the after-ripening.

This type of formula appears to have the practical disadvantage of less stability on storage. After 1 year, the fog was about 1.0 
for emulsions 4-134 and 4-136, and 0.6 for 4-138. Data on the others are not available; the fog may be expected to be less in those with less sulphite. These values could undoubtedly be much reduced by adding bromide before coating.

The experiments completed were all made with deactivated gelatin in order to study as simple a system as practicable. The important practical question of the combination of sulphite with a gelatin having normal activity was not covered. A few data were obtained on the related question of the sulphite content of emulsion gelatins. Three gelatin samples which had recently been tested in the C-1 formula were analyzed for $\mathrm{SO}_{2}$ by the method of Poetschke (34); this procedure, based on weighing $\mathrm{BaSO}_{4}$ derived from oxidation of volatile material by iodine, should be free from positive errors caused by the presence of other compounds in gelatin. The results were, respectively, $0.11,0.15$, and 0.007 percent $\mathrm{SO}_{2}$. Correlation between $\mathrm{SO}_{2}$ content and photographic characteristics of gelatins obviously should not be attempted without a large number of samples, and might then be impossible without analysis for other variables with an accuracy not now available. In the C- 1 formula, the above amounts would correspond to ratios of 10,13 , and $0.3 \times 10^{-3} \mathrm{~g}$ equivalent of $\mathrm{SO}_{2}$ to 1 of $\mathrm{AgBr}$. In this formula, where all the gelatin is added before washing, such amounts of $\mathrm{SO}_{2}$ would be negligible. If, however, gelatin were added after washing, as in many formulas, the ratio with the second of these gelatins might reach $4 \times 10^{-3}$ under conditions which would make its effect quite appreciable. Whether it would be desirable or undesirable would obviously depend on conditions in the emulsion, as it might lead either to increased sensitivity or to the increase in fog and decreased sensitivity characteristic of excessive amounts of sensitizer. Sheppard (35) mentions sulphur dioxide as undesirable in photographic gelatins to which dyes are to be added, but makes no specific reference to any effect on the emulsion. Wentzel (36) and Wall (20) list it as an undesirable impurity, placing the limit at 0.01 percent and 0.1 percent, respectively, without giving reasons for this limitation. The lower limit is to be recommended on the grounds of reproducibility of results with the gelatin, but attention should be called to the possibility of sensitization by amounts which are to be found in emulsion gelatins.

\section{EXPERIMENTS WITH OTHER REDUCING AGENTS}

It is obvious that other reducing agents might be found which would satisfy the condition of forming a molecular compound or complex ion with silver salts. A low rate of reaction is probably a further requirement for sensitization. Experiments were made with only two other materials: hydrazine and sodium arsenite. These were added to portions of an emulsion in amounts sufficient to reduce 1 atom of silver per 1,000 , and digested with it at $55^{\circ}, \mathrm{pH} 6.5,\left[\mathrm{Br}^{-}\right]$ $2 \times 10^{-5}$. The hydrazine produced neither sensitization nor fog in 2 hours. The sodium arsenite in the same time produced an increase over the control which was close to the limit of error. 


\section{CONCLUSIONS}

The data presented in this paper appear to be adequate proof that metallic silver may function as sensitivity nuclei. Silver is very probably formed during digestion with active gelatin; if formed it may increase sensitivity and the results with sulphite indicate, but do not prove, that it does.

The analytical results must be used with some caution but they indicate that the quantity of the nonhalide silver is not satisfactorily related to its sensitizing effect and that, therefore, part of it fails to function as sensitivity nuclei. Chemically equivalent amounts of silver and silver sulphide appear to have roughly the same sensitizing effect. The evidence is not sufficient to decide whether the superior results obtainable with allyl thiocarbamide are to be ascribed to the formation of silver sulphide or to the mechanism of the reaction by which it is formed. Sulphites fall into the same class with the other nuclear sensitizers in that they form a molecular complex with silver salts. Further investigation of reducing agents as sensitizers is desirable to establish how general this rule may be.

The relatively high concentration of sulphite necessary to produce a given rate of reaction, and the marked effect of bromide-ion concentration on the rate, are both in accordance with the low reduction potential of the sulphite. The effect of hydrogen-ion concentration is of the right order of magnitude for any oxidation-reduction reaction.

While many of the emulsions sensitized with sulphite were of commercial quality, none were equal to the best obtainable by standard methods from the same type formulas. The experiments which have been made by no means exhaust the possibilities of improvement. In general, the best results were obtained by rapid after-ripening, with high $\mathrm{pH}$ and low bromide-ion concentration; as there was little tendency to cause fog, the digestion could be carried out in this way, and the emulsion then stabilized by addition of soluble bromide and possibly acid with little loss of sensitivity. The presence of sulphur dioxide in emulsion gelatins assumes a new importance since, under certain circumstances, it may cause sensitization as well as fog.

\section{ACKNOWLEDGMENTS}

C. M. Kretchman has given valuable assistance in the making and testing of all the experimental emulsions.

\section{BIBLIOGRAPHY}

(1) Lüppo-Cramer, Phot. Mitteilungen, p. 328, 1909.

(2) W. Clark, Phot. J., vol. 63, p. 230, 1923.

(3) W. Clark, Phot. J., vol. 64, p. 91, 1924.

(4) W. Clark, Trans. Faraday Soc., vol. 19, p. 309, 1923.

(5) Sheppard, Trivelli, and Loveland, J. Franklin Inst., vol. 200, p. 51, 1925. This article gives an excellent summary of previous work on the subject.

(6) Sheppard, Third Colloid Symposium Monograph, p. 1, 1925.

(7) Sheppard, Phot. J., vol. 65, p. 380, 1925. 
(8) W. Clark, Brit. J. Phot., pp. 227, 243, 1927.

(9) Weigert and Lühr, Zeit. f. wiss. Phot., vol. 27, p. 312, 1930.

(10) Sheppard, Proc. 8th Int. Congress of Phot., p. -

(11) Carroll and Hubbard, B.S. Jour. Research, vol. 8, p. 711, 1932 (RP447).

(12) Carroll and Hubbard, B.S. Jour. Research, vol. 1, p. 56, 1928 (RP20).

(13) Eder, Photographie mit Bromsilber-Gelatine (1903 Ed.), pp. 125 to 140.

(14) Sheppard, Phot. J., vol. 71, p. 313, 1931.

(15) Sheppard, U.S. Patent 1574944.

(16) Sheppard and Punnett, U.S. Patent 1623499.

(17) Trivelli, J. Franklin Inst., vol. 204, p. 649, 1927; vol. 205, p. 211, 1928.

(18) Valenta, Eder's Jahrbuch f. Phot., p. 449, 1894.

(19) Lüppo-Cramer, Phot. Korr., vol. 67, p. 1, 1931.

(20) Wall, Photographic Emulsions, p. 60.

(21) Trivelli and Sheppard, The Silver Bromide Grain of Photographic Emulsions, p. 53.

(22) Sheppard, J. Franklin Inst., vol. 210, p. 587, 1930.

(23) Sheppard and Meyer, Phot. J., vol. 60, p. 17, 1920.

(24) Feigl, Z. f. anal. Chem., vol. 74, p. 369, 1928.

(25) Carroll, Ind. \& Eng. Chem., Analytical Ed., vol. 4, p. 323, 1932.

(26) Carroll and Hubbard, B.S. Jour. Research, vol. 7, p. 811, 1931 (RP376).

(27) Carroll and Hubbard, B.S. Jour. Research, vol. 7, p. 219, 1931 (RP340).

(28) As adopted by the 8th Int. Congress of Phot., 1931.

(29) Davis and Neeland, B.S. Jour. Research, vol. 11, p. 379 (1933) (RP598).

(30) Huse and Meulendyke, Phot. J., vol. 66, p. 306, 1926.

(31) Carroll and Hubbard, B.S. Jour. Research, vol. 10, p. 211, 1933 (RP525).

(32) Carroll and Hubbard, B.S. Jour. Research, vol. 9, p. 529, 1932 (RP488).

(33) Sheppard, Phot. J., vol. 66, p. 399, 1926.

(34) Poetschke, J. Ind. \& Eng. Chem., vol. 5, p. 980, 1913.

(35) Sheppard, Gelatin in Photography, vol. 1.

(36) Wentzel, Fabrikation d. Phot. Platten, Filme u. Papiere (Eder's Handbuch, vol. III, part 1 (1930 edition).

Washington, August 11, 1933. 